\title{
Numerical Studies of Interface Debonding Damage Detection in FRP Strengthened Steel Beam Using Rayleigh Waves
}

\author{
Ying $\mathrm{Xu}^{*}$, Bingcheng Li and Xiaojie Gong \\ ShenZhen Key Lab of Urban \& Civil Engineering Disaster Prevention \& Reduction, Harbin Institute of Technology Shenzhen \\ Graduate School, Shenzhen 518055, China. \\ *Corresponding author
}

\begin{abstract}
This paper studied how to use the variation of the dispersion curves of Rayleigh wave group velocity to detect interfacial debonding damage between FRP plate and steel beam. Since FRP strengthened steel beam is two layers medium, Rayleigh wave velocity dispersion phenomenon will happen. The interface debonding damage of FRP strengthened steel beam have an obvious effect on the Rayleigh wave velocity dispersion curve. The paper first put forward average Euclidean distance and angle separation degree to describe the relationship between the different dispersion curves. Numerical results indicate that there is a approximate linear mapping relationship between the average Euclidean distance of dispersion curves and the length of interfacial debonding damage.
\end{abstract}

Keywords-interfacial debonding damage; rayleigh wave; FRP; dispersion curves; finite element method

\section{INTRODUCTION}

In recent years, scholars of different countries opened a lot of experimental and numerical simulation research in steel structure strengthened design aspects. Among them, related technology research of the CFRP strengthened steel beam has become increasingly perfect[1]. However, the cementing agent between FRP and steel often become the weak link in FRP strengthened steel structure[2] and interfacial debonding damage often happens between FRP and steel beam. In order to ensure the safety and reliability of FRP strengthened steel beam, it is necessary to develop a NDT method to detect the interfacial debonding damage.

$\mathrm{Xu}$ Ying, Liu Zhian etc[3] have put forward to use difference frequency acoustic beam to realize non-contact and long distance detecting for interface debonding damage of FRP strengthened structure. But it has a high demand for the device launching high directivity difference frequency acoustic beam and detection step is too trival.

In recent years, Simon V. Walker etc[4] used nonlinear Rayleigh wave to detect fatigue damage of A36 steel. V. M. Ushakov etc[5] use ultrasonic Rayleigh wave time delay method to detect surface crack. Zhang Jianfeng etc[6] use nonlinear Rayleigh wave to detect the width of the glass surface scratches. Due to the interface debonding damage close to the free surface, using related parameters of Rayleigh wave to detect debonding damage should be feasible.

\section{Principle of Interface Debonding Damage DETECTION}

When apply an excitation on the free boundary surface of the FRP strengthened steel beam, wave and transverse wave will overlap to form Rayleigh wave. Because of the existence of FRP and steel interface debonding damage, Rayleigh wave propagation path changes, which will influence the travel time of Rayleigh wave between two adjacent detection points. Through the change of Rayleigh wave propagation time, it can detect the FRP reinforcement steel beam interface debonding damage. Detecting process is shown in figure 1.

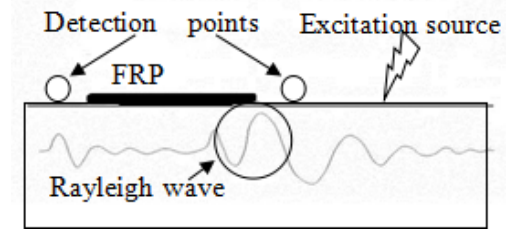

FIGURE I. DETECTION PRINCIPLE USING RAYLEIGH WAVE

When the debonding damage occurs at the end of the FRP plate, Rayleigh wave will propagate in the steel to the right end of debonding damage as shown in figure 1 and then propagate in the two layers medium of FRP and steel. When debongding damage occurs in middle of FRP strengthened steel beam, Rayleigh wave first propagates in multilayer medium. After propagate to debonding damage, most of the Rayleigh wave decomposes body wave and continues to propagate in the steel. Only a little Rayleigh wave converts into Lamb wave mode and continues to propagate in the FRP[7]. Anyway, the velocity reducing amplitude of Rayleigh wave between two detection point and the total length of debonding damage has a strong linear mapping relation. In the paper, the excitation source has a center frequency. The selection of excitation frequency should consider steel beam section height, so as to make sure that the center Rayleigh wave length is less than one third of the steel beam section height.

\section{Debonding Damage Detection Methods}

Detecting the length of debonding damage according to Rayleigh wave group velocity dispersion curve should construct a function and make the function value and interface 
debonding damage length have a stable mapping relation. In the actual detection, we can detect debonding damage length by calculating the function value. In this paper, we use two kinds of distance function. That is the Euclidean distance and Angle separation degree. The functions are defined as follows:

$$
\begin{aligned}
& d_{\mathrm{E}}=\sqrt{\sum_{i=1}^{n}\left(v_{\mathrm{h} i}-v_{\mathrm{d} i}\right)^{2}} \\
& d_{\mathrm{S}}=\frac{\sum_{i=1}^{n} v_{\mathrm{h} i} \times v_{\mathrm{d} i}}{\sqrt{\sum_{i=1}^{n} v_{\mathrm{h} i}^{2} \sqrt{\sum_{i=1}^{n} v_{\mathrm{d} i}^{2}}}}
\end{aligned}
$$

where, $d_{\mathrm{E}}$ Euclidean distance;

$d_{\mathrm{S}}$ Angle separation degree;

$v_{\mathrm{h} i}$ Rayleigh wave velocity corresponding to the ith kind frequency of health structure;

$v_{\mathrm{d} i}-$ Rayleigh wave velocity corresponding to the ith kind frequency of the structure to be measured.

This paper put forward normalized average Euclidean distance. The normalized average Euclidean distance can reflect the damage degree of FRP strengthened steel beam effectively. The average Euclidean distance and normalized average Euclidean distance are defined as follows.

$$
\begin{gathered}
d_{\text {Eaverage }}=\sqrt{\sum_{i=1}^{n}\left(v_{\mathrm{h} i}-v_{\mathrm{d} i}\right)^{2}} / n \\
d_{\text {EAnorm }}=\frac{d_{\text {Eaverage }}}{d_{\text {EA max }}-d_{\text {EA min }}}
\end{gathered}
$$

where, $d_{\text {Eaverage }}$ Average Euclidean distance;

$$
\begin{aligned}
& d_{\text {EA max }}-\text { The maximum average Euclidean distance; } \\
& d_{\text {EA min }}-\text { The minimum average Euclidean distance; } \\
& d_{\text {EAnorm }}-\text { Normalized average Euclidean distance. }
\end{aligned}
$$

We can use average Euclidean distance to calculate the length of interface debonding damage. The Calculation formula is as follows:

$$
L_{\text {Debonding }}=d_{\text {EAnorm }} \times L_{\mathrm{FRP}}
$$

Where,

$$
\begin{gathered}
L_{\mathrm{FRP}}-\text { FRP plate length; } \\
L_{\text {Debonding }}-\text { Debonding damage length. }
\end{gathered}
$$

\section{DEBONDING DAMAGE DETECTION EXAMPLE}

\section{A. The Establishment of the FRP Strengthened Steel Beam 3D Numerical Model}

1) Unit and Grid: The simulation of FRP and steel adopts low-order entity unit. Unit quality distributes to nodes evenly, which is suitable for numerical simulation of wave propagation. When simulate the wave propagation, the grid size should be less than one over twenty of the wavelength.

2) The Actual Effect of Damping: Damping not only let amplitude attenuation, but also affects the phase Angle. In this paper, we adopt material damping coefficient. The conversion relation of material damping coefficient and viscous damping ratio is showed as the following formula.

$$
\xi=\frac{\varsigma}{\pi f}
$$

where, $\xi$-Material damping coefficient;

$$
\zeta-\text { Viscous damping ratio; }
$$

$\mathrm{f}$-Excitation frequency.

3) Sampling Frequency: Using New mark time integration method, the integral time step $\Delta \mathrm{t}$ can be determined by the following formula ${ }^{[8]}$.

$$
\Delta t \leq \frac{1}{20 f_{\max }}
$$

where, fmax_—Effective highest frequency.

4) Numerical Simulation of the Interface Debonding Damage: For FRP and steel debonding damage simulation, this paper does not consider the effect of cementation layer to Rayleigh wave propagation. For the area where FRP and steel doesn't debond, the nodes of FRP and steel couple. Thus, in the interface the displacement of the nodes of FRP and steel is always consistent when Rayleigh wave propagates. For the area where FRP and steel debond in the interface, the node of FRP and steel not couple and the node displacement are relatively independent.

5) Boundary Processing: In order to save computation time, the paper applies viscoelastic absorbing boundary on both ends of the limited finite element model to simulate the Rayleigh wave propagation. Viscoelastic absorbing boundary are realized through the spring - damper unit, as shown in figure 2. The spring coefficient and damping coefficient of the three 
dimensional artificial viscoelastic boundary is defined as follows[9].

$$
\begin{gathered}
K_{\mathrm{BT}}=0.5 \frac{G}{r_{\mathrm{b}}} A, C_{\mathrm{BT}}=\rho c_{\mathrm{s}} A \\
K_{\mathrm{BN}}=\frac{G}{r_{\mathrm{b}}} A, C_{\mathrm{BN}}=\rho c_{\mathrm{s}} A
\end{gathered}
$$

where, $A-$ Function area of boundary area.

$K_{\mathrm{BT}} \longrightarrow$ Tangential stiffness coefficient;

$C_{\mathrm{BT}} \longrightarrow$ Tangential damping coefficient;

$K_{\mathrm{BN}} \longrightarrow$ Normal stiffness coefficient;

$C_{\mathrm{BN}} \longrightarrow$ Normal damping coefficient.

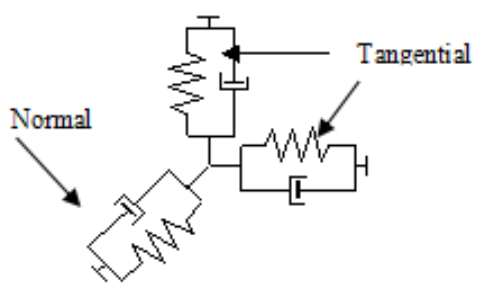

FIGURE II. SCHEMATIC OF THREE-DIMENSIONAL ARTIFICIAL VISCOELASTIC BOUNDARY

\section{B. Rayleigh Wave Cross Correlation Analysis}

Rayleigh wave group velocity between the two detection point before and after damage is calculated by the following formula:

$$
V_{\mathrm{R} i}=\Delta x / \Delta t_{i}
$$

where, $\Delta t_{i}$ Rayleigh wave delay time; $\Delta x-$ Two detection point spacing.

Rayleigh wave delay time can be calculated by cross correlation analysis. The correlation coefficient of two Rayleigh wave packet can calculate by the following formula.

$$
R_{21}(\tau)=\frac{1}{N} \sum_{n=1}^{N} v_{1}(n) v_{2}(n+\tau)
$$

where, $\tau$ - time delay;

$v_{1}(n)-$ Vertical displacement time history curve from the detection point 1

$v_{2}(n+\tau)-$ Vertical displacement time history curve from the detection point 2

$$
R_{21}(\tau) \longrightarrow \text { Correlation coefficients }
$$

The maximum correlation coefficient value is the delay time of Rayleigh wave.

\section{Not All Cross FRP Strengthened Steel Beam Debondding Damage Detection}

This paper simulated three different kinds of debonding damage, including one side debonding, two sides debonding, the middle debonding and two sides debonding occurring at the same time. Under different working conditions, comparing the Rayleigh wave group velocity dispersion curves of the damage structure and the health structure, we can detect the corresponding debonding damage length.

Figure 3 shows the difference of Rayleigh wave group velocity dispersion curves between one side debonding structure and health structure. From the figures, we can know Rayleigh wave group velocity dispersion curve shape of different debonding damage length is similar. But with the debonding damage length increasing, Rayleigh wave group velocity dispersion curve will move down parallel. If calculate the Euclidean distance and Angle separation degree of the Rayleigh wave group velocity dispersion curves of structure with different debonding damage lengths, the Angle separation degree is close to 1 and Euclidean distance increases as the debonding damage length increases. But there is no pure linear relationship between Euclidean distance and debonding damage length. Due to the dispersion curve is close to parallel, Angle separation degree is close to 1. So the Angle separation degree can be used to judge whether the information collected by the detector is accurate.
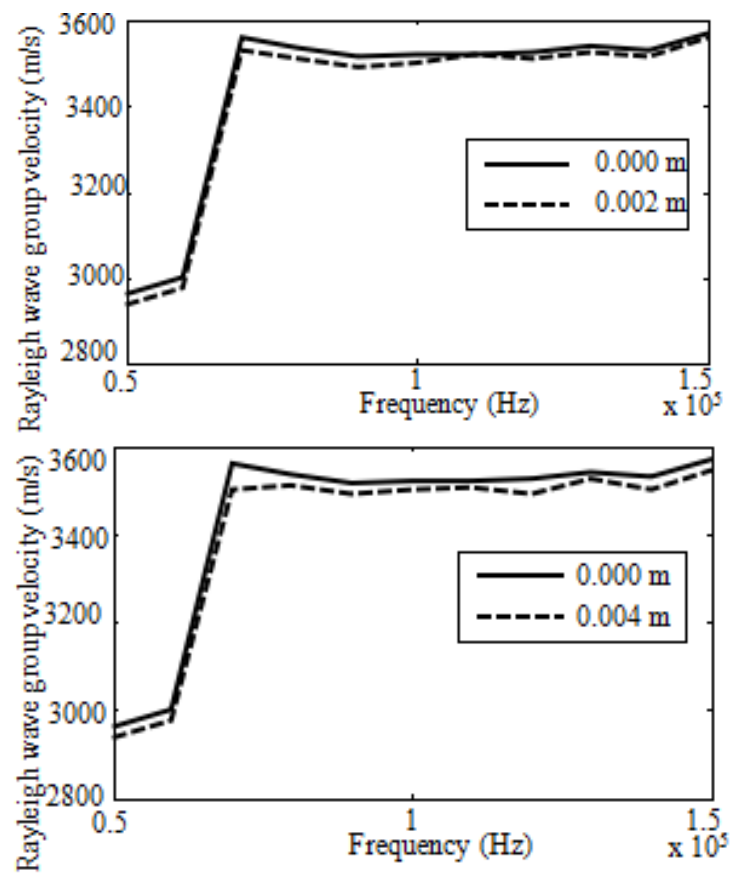


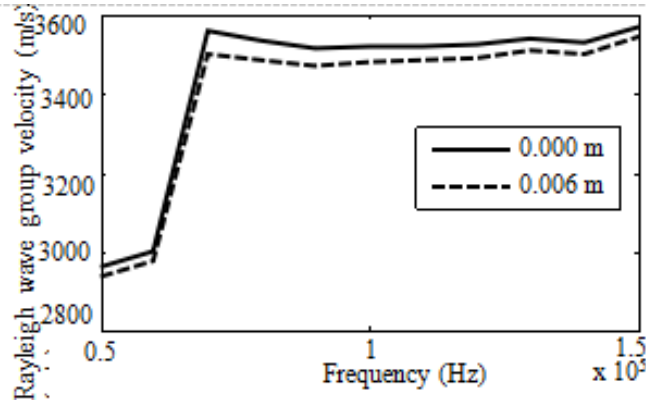

FIGURE III. COMPARISON OF DISPERSION CURVES UNDER DIFFERENT LENGTHS OF UNILATERAL PLATE END DEBONDING

The comparison results of Rayleigh wave group velocity dispersion curve between different debonding damage strurure and health structure are shown in table 1, table 2 and table 3. From these tables, we can know, no matter the interface debonding damage appeared in the plate side or middle, the normalized average Euclidean distance and interface debonding damage length have approximate linear mapping relationship. Deduced debonding damage length from the normalized average Euclidean distance is very accurate and the error is less than $2 \mathrm{~cm}$. So use normalized average Euclidean distance to detect interface debonding damage of FRP strengthened steel beam is feasible.

TABLE I. COMPARISON RESULTS OF DISPERSION CURVES UNDER DIFFERENT ONE SIDE DEBONDING LENGTH

\begin{tabular}{ccc}
\hline $\begin{array}{c}\text { One side } \\
\text { debonding } \\
\text { length (m) }\end{array}$ & $\begin{array}{c}\text { Normalized average Euclidean } \\
\text { distance }\end{array}$ & $\begin{array}{c}\text { Detected damage } \\
\text { length (m) }\end{array}$ \\
\hline 0.02 & 0.113 & 0.034 \\
0.04 & 0.160 & 0.048 \\
0.06 & 0.206 & 0.062 \\
0.08 & 0.274 & 0.082 \\
0.10 & 0.320 & 0.096 \\
\hline
\end{tabular}

TABLE II. COMPARISON RESULTS OF DISPERSION CURVES UNDER DIFFERENT TWO SIDES DEBONDING LENGTH

\begin{tabular}{llc}
\hline $\begin{array}{l}\text { Two sides } \\
\text { debonding } \\
\text { length }(\mathbf{m})\end{array}$ & $\begin{array}{c}\text { Normalized average Euclidean } \\
\text { distance }\end{array}$ & $\begin{array}{c}\text { Detected damage length } \\
(\mathbf{m})\end{array}$ \\
\hline $2 \times 0.02$ & 0.175 & 0.053 \\
$2 \times 0.04$ & 0.311 & 0.093 \\
$2 \times 0.06$ & 0.407 & 0.122 \\
$2 \times 0.08$ & 0.539 & 0.162 \\
\hline
\end{tabular}

TABLE III. COMPARISON RESULTS OF DISPERSION CURVES UNDER DIFFERENT TOTAL LENGTH OF TWO SIDES. DEBONDING AND MIDSPAN DEBONDING

\begin{tabular}{ccc}
\hline $\begin{array}{c}\text { Total debonding } \\
\text { length }(\mathbf{m})\end{array}$ & $\begin{array}{c}\text { Normalized average } \\
\text { Euclidean distance }\end{array}$ & $\begin{array}{c}\text { Detected damage } \\
\text { length }(\mathbf{m})\end{array}$ \\
\hline $2 \times 0.02+0.02$ & 0.267 & 0.071 \\
$2 \times 0.02+0.04$ & 0.312 & 0.094 \\
\hline
\end{tabular}

For FRP strengthened steel beam, regardless of the interface debonding damage appears in the plate side or middle, the effectiveness of the FRP plate strengthened structure and whether tle failure will happen is decided by not dabongding FRP plate length. The calculation formula is as following:

$$
L_{\text {NDebonding }}=\left(1-d_{\text {EAnorm }}\right) \times L_{\mathrm{FRP}}
$$

where, $L_{\text {NDebonding }} \longrightarrow$ Not debonding FRP plate length.

When, $L_{\text {NDebonding }}<L / 2+L_{\text {effective }}$, where $L_{\text {effective }}$ is one side effective bond length of FRP plate [12]. It can conclude that the FRP plate strengthening lacks of ductility and the ultimate bearing capacity contributed by FRP plate has been lower than design value, where.

\section{CONCLUSION}

This paper put forward to a new method to detect the interface debonding damage of FRP strengthen steel beam by dispersion property of Rayleigh wave. This method uses average Euclidean distance between Rayleigh wave group velocity dispersion curve of interface debonding damage structure and health structure to calculate the debonding length. In order to implement this detection method, debonding damage detection method based on the numerical model of structure is presented. The not full cross FRP strengthened steel debonding damage detection are simulated by the finite element The numerical simulation results prove that the new method to detect debonding damage of FRP strengthened steel beam is feasible.

\section{ACKNOWLEDGMENT}

Funding of NSFC (51278156) and basic project of Shenzhen Science \& Technology Program (JCYJ201407041 72618811).

\section{REFERENCES}

[1] Cheng Jiangmin, Cheng Bo, Qiu He, et al. Research development of steel structure reinforcement[J]. Steel Construction, 2012,11:3.

[2] Zheng Yun, Ye Lieping, Yue Qingrui, et al. Progress in research on steel structures strengthened with FRP[J]. Industrial Construction, 2005, 35(8) 20-25.

[3] Liu Zhian. Numerical simulation for romote acoustic excitation based NDT method of interfacial damage in FRP-reinforced structures[D]. Harbin: Harbin Institute of Technology, 2013: 34-50.

[4] Walker S V, Kim J, Qu J, et al. Fatigue damage evaluation in A36 steel using nonlinear Rayleigh surface waves[J]. NDT \& E International, 2012 48: $10-15$.

[5] Ushakov V M, Davydov D M, Domozhirov L I. Studying the growth kinetics of fatigue cracks using the method of ultrasonic Rayleigh waves[J]. Russian Journal of Nondestructive Testing, 2012, 48(6): 323-329.

[6] Zhang J, Xuan F, Yang F. Effect of surface scratches on the characteristics of nonlinear Rayleigh surface waves in glass[J]. Journal of Non-Crystalline Solids, 2013, 378: 101-105.

[7] Chakrapani S K, Dayal V, Dunt J. Rayleigh wave interaction and mode conversion in a delamination[C]. New York: AIP Publishing, 2014, 1581(1): 690-696.

[8] Moser F, Jacobs L J, Qu J. Modeling elastic wave propagation in waveguides with the finite element method[J]. Ndt \& E International, 1999, 32(4): 225-234. 
[9] Deeks A J, Randolph M F. Axisymmetric time-domain transmitting boundaries[J]. Journal of Engineering Mechanics, 1994, 120(1): 25-42.

[10] Godoy E, Durán M, Nédélec J. On the existence of surface waves in an elastic half-space with impedance boundary conditions[J]. Wave Motion, 2012, 49(6): 585-594.

[11] Zhang Y, Xu Y, Xia J. Wave fields and spectra of Rayleigh waves in poroelastic media in the exploration seismic frequency band[J]. Advances in Water Resources, 2012, 49: 62-71

[12] Nozaka K, Shield C K, Hajjar J F. Effective bond length of carbon-fiber-reinforced polymer strips bonded to fatigued steel bridge I-girders[J]. Journal of Bridge Engineering, 2005, 10(2): 195-205. 